\title{
User Adaptation to Constant Change in Algorithmically-Driven Social Platforms
}

\author{
Michael A. DeVito \\ Northwestern University \\ Evanston, IL 60208, USA \\ devitom@u.northwestern.edu
}

\section{ABSTRACT}

Social platforms present a challenge for self-presentation and identity management by obscuring audiences behind algorithmic mechanisms. Users are increasingly aware of this and actively adapting through folk theorization, but we do not know how users are coping with the constant change endemic to these platforms, or how we can assist users in this coping process. My dissertation will examine how users perceive and adapt to the constantly-changing platform space using self-presentation and audience management as an illustrative case.

\section{CCS CONCEPTS}

- Human-centered computing $\rightarrow$ Empirical studies in $\mathbf{H C l}$; $•$ Social and professional topics $\rightarrow$ User characteristics

\section{KEYWORDS}

Algorithms; folk theories; social platforms; adaptation; change; software updates

Permission to make digital or hard copies of part or all of this work for personal or classroom use is granted without fee provided that copies are not made or distributed for profit or commercial advantage and that copies bear this notice and the full citation on the first page. Copyrights for third-party components of this work must be honored. For all other uses, contact the Owner/Author.

CHI'19 Extended Abstracts, May 4-9, 2019, Glasgow, Scotland UK

(C) 2019 Copyright is held by the owner/author(s).

ACM ISBN 978-1-4503-5971-9/19/05.

https://doi.org/10.1145/3290607.3299082 


\section{ACM Reference format:}

Michael A. DeVito. 2019. User Adaptation to Constant Change in Algorithmically-Driven Social Platforms. In CHI Conference on Human Factors in Computing Systems Extended Abstracts (CHI'19 Extended Abstracts), May 4-9, 2019, Glasgow, Scotland, UK. ACM, New York, NY, USA. 6 pages. https://doi.org/10.1145/3290607.3299082

\section{CONTEXT AND MOTIVATION}

Imagine that you have achieved a dream: living off the profits from selling your artwork online via Etsy storefront. You've read the protips blogs, talked to other store owners, and experimented on your own. Over time, you've created a strategy to keep your art visible in this vast marketplace by appealing to the product search algorithm via embedding specific keywords and re-posting fresh listings every few days. Then, one day, your search ranking drops, and profits dry up. Etsy customer service tells you nothing can be done; they can't tell you what changed in their proprietary search algorithm.

Imagine that you are a closeted bisexual, nonbinary individual from a highly religious family, striking out on your own. The local queer Facebook community becomes your lifeline. It provides you with leads on housing, jobs, and importantly: friends that validate your core identity. At the same time, while they may not be so supportive, your family is still important to you. Mediating your relationship with them via Facebook provides just the right mix of contact and distance to avoid conflict. You've taken the advice of your new friends and a few relevant blogs, and keep your queer-coded content to closed groups, where you know the News Feed algorithm won't distribute the content to non-members. Then, one day, Facebook delivers some helpful suggestions of new groups to join to your not-so-accepting grandmother; about half of them are queer groups from your new city. Facebook did not mean to out you, but an algorithmic change designed to help people close to you find relevant groups to join brought your membership in the local queer community to light. Grandma is not happy.

These hypothetical users were aware that the social platform they relied on for both benefit and protection were algorithmically-driven, and that accounting for this algorithm could be beneficial. Implicitly, they knew that to achieve their goals while avoiding pitfalls ranging from embarrassment [11] to stigmatization and physical threats [4], users of social platforms with algorithmically-driven content delivery and discovery systems (e.g., Facebook's News Feed, YouTube's recommendations) must decide how to use the visibility and audience management tools platforms afford them as part of the self-presentation process $[1,11]$.

Despite the fact that these kinds of systems are often opaque, making audiences difficult to predict or reliably target [1], these users persisted. They formed their own folk theories, or lay, socially-constructed conceptions of how a platform works, to guide their subsequent on-platform behavior [2,3] and help in the construction of a self-presentation strategy. They did their due diligence - but then, the platforms changed how their algorithms operate. Constant tweaks to content distribution algorithms mean constant tweaks to the environment users are making selfpresentation decisions in. The platform landscape is an ever-shifting environment [6] with few 
cues for users as to how and when to adapt their behavior - leaving some of even the most diligent users and their strategies behind.

Still, despite the opaque nature of these algorithmically-driven systems, and the limited-cues environment that results, users do appear to adapt, and even resist and appropriate changes in heightened situations (e.g., [3]). For many of these users, this is an absolute necessity - social platforms, acting as the modern equivalent of a public square for individuals and businesses alike [7], have become deeply integrated into their personal and professional lives (e.g. [4, 5, 10]), even to the level of discrete social processes (e.g., [2]). Simply abandoning platforms is not an option for these users, despite the anxiety that working within a constantly-changing algorithmically-driven system can prompt [9]. Due to the importance, visibility, and centrality of these platforms in our society [7], users must adapt. However, our current understanding of this kind of algorithmic adaptation is limited to accounts of initial adaptation to algorithmic systems (e.g., [10]) and responses to highly visible changes with accompanying public controversies (e.g., [3]). We do not yet have a detailed understanding of the everyday adaptations that users may make to keep up with rapid and sometimes subtle changes to these systems - the kinds of changes, and associated challenges, that our two friends from above now face. In my dissertation work, I will explore user adaptation strategies over time around changing algorithmic platforms using self-presentation and audience management as an illustrative case.

My dissertation aims to provide guidance to platform designers and operators as to how to keep users better informed and ease transitions to new versions of algorithmically-driven systems, as well as provide a deeper understanding of how user algorithmic literacy, currently treated as a static concept [10], evolves over time as systems change. In doing so, I also hope to extend the existing literatures on audience management, self-presentation, and algorithmic understanding/explanation.

\section{CURRENT QUESTIONS}

My prior work suggests that that the formation and updating of folk theories is well-integrated into self-presentation [2]. Additionally, these folk theories are reactive to information both endogenous (e.g., interface changes) and exogenous (e.g., news stories, rumors) [2,3] to the platform in question. Earlier work on updating information systems in organizational contexts with planned changes also suggests that adaptation to technological change is dependent on the kind of environment the users perceive and how this environment frames change [1, 12]. My follow-up work suggests that this structure may translate to the much less prescriptive environment of social platforms [3]. Given this, to better understand how users adapt to algorithmic change over time, I must first understand the change environment that users perceive and how both platform-endogenous and -exogenous information frames change. This includes better understanding both the information environment itself and how users are perceiving it. As such, I ask:

RQ1: What changes, and at what frequency, do users perceive as being made to leading algorithmically-driven social platforms? 
RQ2: How are changes to leading algorithmically-driven platforms framed by official and popular unofficial sources?

RQ3: To what extent do users perceive the platform environment as unstable?

Answering these questions will provide a better understanding of the change environment, which in turn will provide a basis for investigating user reactions to said environment. With preliminary answers to RQs $1-3$ in mind, I will then ask:

RQ4: How do users adapt to a changing platform environment when making audience management and self-presentation decisions?

\section{WORK IN PROGRESS: PRELIMINARY STUDIES}

Emerging results from two preliminary studies will provide guidance on user adaptations to look for, how adaptation-related information diffuses, and which levers of change to test in my dissertation.

One study, currently in the analysis phase, presents users with new information about changes to Facebook from multiple sources, and assesses change in the user's folk theories of the platform. Preliminary analysis suggests that news content is especially relevant to changes in user folk theories of mechanism, pointing to the need to closely track platform-related news content and exposure during my dissertation studies.

The other study is a qualitative and network analysis-based exploration of recent attempts to roll back Twitter's algorithmic timeline via the use of keyword muting. This study will provide insight into how adaptations originate among power users, and how they might diffuse to normal users of Twitter. Understanding diffusion of adaptations is crucial to understanding key differences between change in traditional organizations and in the distributed landscape of social platforms, informing later sampling.

\section{NEXT STEPS: THE DISSERTATION}

My dissertation consists of two linked studies to capture perceptions of the changing platform environment alongside adaptation behaviors. Study one captures the change, real or perceived, in the platform environment, while study two uses this data to inform a dive into user adaptation behaviors.

I will address RQs 1 and 2 by analyzing information about change across a selection of major platforms over the period of a year. Sources include statements and news stories on platform change, as well as relevant financial and patent filings. To catch user-perceived changes that may not be highlighted in these sources, I will employ online ethnographic techniques to collect data on "folk rumoring" about platform changes, such as the intense discussions about changes to platform algorithms that regularly take place on each platform's respective subreddit. This will provide a first accounting of platform change over time, and also set up externally-valid, real-time prompts for use in study two. By the DC, I will have the first six months' worth of data from this study for analysis and discussion. 
To address RQ3, study two will employ a mixed-methods user survey to investigate the environment constant platform change has created. The survey will include open-ended items which ask users about their self-presentation and audience management tactics, their folk theories of platform algorithms, and their perceptions of the amount, type, and frequency of change in these systems. These will be compared to items which measure key concepts around adaptation behavior and the effects of a constantly-changing environment, drawn from $\mathrm{HCl}$-related literature on technological change (e.g., personality traits, predisposition to innovate, computer self-efficacy [13]) and psychological literature on changing environments (e.g., emotional/psychological resilience, anxiety/burnout levels [8]).

To address RQ4 and better understand how adaptation processes play out behaviorally, study three will employ a six-month user diary study overlapping with study one. The diary will consist of prompts which ask participants how they are enacting self-presentation and audience management, what their current folk theory of the platform is, as well as their perceptions of and reactions to changes being tracked by study one. This study will end with in-depth interviews, allowing follow-up on previous responses. I will enroll 60 participants in three comparative groups: "average" users, users with heightened self-presentation concerns (e.g., LGBTQ+ individuals), and users for whom self-presentation is a job (e.g., Etsy storefront owners, Instagram influencers). By comparing these groups, I will be able to create a robust accounting of how adaptation processes vary among users along with their self-presentation goals, and generate design and theoretical implications with broad applicability.

\section{ACKNOWLEDGEMENTS}

This work is partially supported by the US National Science Foundation under grant IIS-1617387. It is also partially supported by the Northwestern University Graduate School, under the Graduate Research Grant program. The author acknowledges valuable feedback and support from Jeremy Birnholtz, Ashley Walker, and Julia Fernandez, as well as the combined support of the undergraduate RA teams that have been indispensable to the prior work this study builds on.

\section{REFERENCES}

[1] Michael A. DeVito, Jeremy Birnholtz, and Jeffrey T. Hancock. 2017. Platforms, People, and Perception: Using Affordances to Understand Self-Presentation on Social Media. In Proceedings of CSCW 2017, 740-754.

[2] Michael A. DeVito, Jeremy Birnholtz, Jeffrey T. Hancock, Megan French, and Sunny Liu. 2018. How People Form Folk Theories of Social Media Feeds and What It Means for How We Study Self-Presentation. In Proceedings of CHI 2018

[3] Michael A. DeVito, Darren Gergle, and Jeremy Birnholtz. 2017. "Algorithms ruin everything": \#RIPTwitter, Folk Theories, and Resistance to Algorithmic Change in Social Media. In Proceedings of CHI 2017, 3163-3174.

[4] Michael A. DeVito, Ashley M. Walker, and Jeremy Birnholtz. 2018. "Too Gay for Facebook:" Presenting LGBTQ+ Identity Throughout the Personal Social Media Ecosystem. Proceedings of the ACM on Human-Computer Interaction, 2, CSCW: 44.

[5] Nicole B Ellison, Charles Steinfield, and Cliff Lampe. 2007. The benefits of Facebook "friends:" Social capital and college students' use of online social network sites. Journal of Computer-Mediated Communication, 12, 4: 1143-1168

[6] Tarleton Gillespie. 2014. The Relevance of Algorithms. In Media technologies: Essays on communication, materiality, and society, Tarleton Gillespie, Pablo Boczkowski and Kirsten Foot (ed.). MIT Press, Cambridge, MA, 167-193. 
[7] Tarleton Gillespie. 2018. Platforms are not intermediaries. Georgetown Law Technology Review, 2, 2: 198-216.

[8] Veronica Greco and Derek Roger. 2001. Coping with uncertainty: The construction and validation of a new measure. Personality and individual differences, 31, 4: 519-534.

[9] Shagun Jhaver, Yoni Karpfen, and Judd Antin. 2018. Algorithmic Anxiety and Coping Strategies of Airbnb Hosts. In Proceedings of 2018 CHI Conference on Human Factors in Computing Systems, 421.

[10] Erin Klawitter and Eszter Hargittai. 2018. "It's Like Learning a Whole Other Language:" The Role of Algorithmic Skills in the Curation of Creative Goods. International Journal of Communication, 1221.

[11] Eden Litt, Erin Spottswood, Jeremy Birnholtz, Jeff T Hancock, Madeline E Smith, and Lindsay Reynolds. 2014. Awkward encounters of an other kind: collective self-presentation and face threat on facebook. In Proceedings of CSCW 2014, 449-460.

[12] Wanda J Orlikowski and Debra C Gash. 1994. Technological frames: making sense of information technology in organizations. ACM Transactions on Information Systems (TOIS), 12, 2: 174-207.

[13] Kurt Schmitz, James TC Teng, and Kimberly Webb. 2016. Capturing the Complexity of Malleable IT Use: Adaptive Structuration Theory for Individuals. MIS Quarterly, 40, 3: 663-686. 